\title{
Two routes to closure: Time pressure and goal activation effects on executive control
}

\begin{abstract}
In the present study the impact of need for cognitive closure (NFC) manipulations via time pressure and explicit closure goal activation on executive control was investigated. Although there is some evidence that NFC, measured as an individual variable, is related to better performing in attentional tasks involving executive control, these results have never been validated across different manipulations of NFC. Thus, in the present study we induced NFC via internal and external time pressure and tested the impact of these manipulations on the performance in tasks that measure executive control, i.e., the Stroop and switching tasks. The results revealed that induced high (vs. low) NFC, indeed boosted performance in executive control tasks. Moreover, there was no difference in the effect of both NFC manipulations on task performance. The implications regarding the role of executive control and specific NFC manipulations in social cognition are discussed.
\end{abstract}

Key words: need for closure, time pressure, goal activation, executive control

The results of previous studies demonstrated that the need for cognitive closure (NFC), defined as a need to have any answer on a given topic, as opposed to further ambiguity (Webster \& Kruglanski, 1994) corresponds with individual differences in the ability to handle interference (Kossowska, 2007a; Kossowska, Orehek \& Kruglanski, 2010). In the present study we validated this finding using NFC manipulations via time pressure and goal activation. We focused specifically on these two types of NFC inductions, as previous results suggested, that they may not only affect epistemic motivation, but also influence other aspects of knowledge formation, in particular cognitive capacity (Bukowski, von Hecker \& Kossowska, 2013).

\section{Individual differences in need for closure and executive} control

NFC has been described as the tendency to reduce the feeling of discomfort experienced in the face of cognitive uncertainty through quick formulation of a hypothesis and its short validation (Webster \& Kruglanski, 1994). Individuals high in dispositional NFC are characterized by a preference for order and for predictability in their lives, afforded by secure and stable knowledge that is reliable across circumstances and unchallenged by exceptions. High NFC individuals also experience an urgent desire to reach swift and firm decisions, reflected in their need for decisiveness, and they feel discomfort with ambiguity, experiencing situations lacking closure as aversive. Finally, they are closed-minded, resistant to information inconsistent with their firm opinions, and reluctant to have their knowledge challenged. It is also well documented that the cognitive processes used by high-need-for-closure individuals to reduce uncertainty are category-based, nonsystematic, and heuristic. In contrast, individuals with low levels of need for closure prefer to reduce uncertainty by using piecemeal or individuation processes. This preference is manifested in vigilant behavior that is based on a systematic and effortful search for relevant information, its evaluation, and its unbiased assimilation (Kruglanski, Dechesne, Orehek, \& Pierro, 2009; Bar-Tal, Kishon-Rabin, \& Tabak, 1997; Driscoll, Hamilton, \& Sorrentino, 1991).

The previous research allowed one to conclude that the tendency of individuals high in the NFC to focus or 'freeze' on a specific region of the cognitive field (i.e. on specific categories, or concepts) represents a compensatory mechanism developed in order to make up for their cognitive-capacity limitations, and affording superior cognitive selectivity and the ability to shut out irrelevant distractions and noise (Kossowska, 2007a,b; Kossowska et al., 2010). They also revealed that NFC

\footnotetext{
* Jagiellonian University

** Institute of Psychology, al. Mickiewicza 3, 31-120 Kraków, Poland; malgorzata.kossowska@uj.edu.pl
} 
reduces the incidence of uncertainty, conflict, and error because it motivates to achieve closure that successfully accommodate experience, results in zealous goal pursuit that narrows attention away from discrepancy, or provides rigid predictions that assimilate inconsistent observations (Kossowska et al., 2010; Amodio, Jost, Masters \& Yee, 2008; Inzlicht, McGregor, Hirsh \& Ash, 2009).

These are important findings as efficient control processes are essential in all situations in which a clear and stable picture has to be distilled from a fuzzy and complex stimulus configuration (e.g. Dijksterhuis, Van Knippenberg, Kruglanski, \& Schaper, 1996). Executive control is responsible for stopping reactions on new, conflicting, or irrelevant information, i.e., which could violate coherence and stability of the knowledge system, from further processing. By reducing the probability that contradictory, new or inconsistent information is encoded, perception is guided effectively toward a relatively clearcut, unambiguous impression, judgment, or opinion (Miller \& Cohen, 2001; Miyake, Friedman, Emerson, Witzki, \& Howerter, 2000). Thus, executive control may be seen as process responsible for different social effects related to NFC, as, among others, impression primacy effect (Kruglanski \& Freund, 1983; Freund, Kruglanski, \& Schpitzaijzen, 1985; Heaton \& Kruglanski, 1991; Webster \& Kruglanski, 1994; Webster, Richter, \& Kruglanski, 1996), the tendency to base judgments on prevalent stereotypes (Dijksterhuis et al., 1996; Jamieson \& Zanna, 1989), and to assimilate numerical estimates to anchor values (Kruglanski \& Freund, 1983), increases overattribution bias (Webster, 1993a), mere exposure effects (Kruglanski, Freund, \& BarTal, 1996) or the effects of priming (e.g. Ford \& Kruglanski, 1995).

\section{Need for closure induction and executive control}

NFC usually treated as individual characteristic, it is also seen as a motivational tendency that may be induced situationally (Kruglanski \& Webster, 1996). A most straightforward instigator of high NFC is time pressure (e.g., Kruglanski \& Freund, 1983, Richter \& Kruglanski, 1998; Roets \& Van Hiel, 2011). However, various situations wherein information processing is difficult, laborious, or aversive can also result in a heightened desire for closure. For example, if the task at hand is dull or of low interest (e.g., Webster, 1993a), if performance is impeded by external stressors such as environmental noise (e.g., Kruglanski, Webster, \& Klem, 1993), or if processing is felt to be laborious due to fatigue (e.g., Webster et al., 1996) or intoxication (e.g., Webster, 1993b), people may also experience an increased desire to reach closure (see Kruglanski, 2004, Kruglanski \& Webster, 1996, for an overview).

Although the effects of experimentally induced NFC have been repeatedly cross-validated with converging effects of individual differences in dispositional NFC, and vice versa (e.g., Chiu, Morris, Hong, \& Melon, 2000; Roets, Van Hiel, Cornelis, \& Soetens, 2008; Webster \& Kruglanski, 1994), the influence of situationally induced NFC on information processing in the task involving executive control was never tested. Moreover, these cross-validations do not preclude the possibility that some particular NFC manipulations do not only affect epistemic motivation, but also influence other aspects of knowledge formation, in particular cognitive capacity thus influence on the ability to handle interference.

On the one hand, Roets et al. (2008) found that in addition to their motivational effect on the extent of information sampling, both noise and time pressure also affected performance in the easy versions of the task. The researchers suggest that noise and time pressure manipulations indeed yield a "double effect" on both motivation and cognitive capacity. On the other hand, Bukowski and colleagues (2013) demonstrated that the type of NFC manipulation (by external vs internal time constraints) has a differential impact on the two aspects of knowledge creation: consistency of currently processed information with background knowledge and timing of presentation of information, while reasoning about social relations. That research revealed, that externally (environmentally) imposed time pressure accompanied by difficult task conditions (e.g., late presentation of diagnostic information that imposes a load on working memory) resulted in a tendency to prematurely terminate the reasoning process and to form more erroneous representations. Impaired reasoning in external time pressure conditions should therefore be related to cognitive demands imposed by the reasoning task itself. However, if cognitive closure motive was activated via internal standards (by emphasizing the value of quick vs. accurate reasoning strategies), the reliance on information consistent with background knowledge was a predominant processing strategy. These results suggest the functional differences of these two types of NFC manipulations. Specifically, these results revealed that the time pressure manipulation created an external constraint that affected specifically the speed of information processing and thus also impaired the ability of participants to process the information presented late in the sequence. In contrast, the NFC goal activation manipulation activated a representation of the task in which the task was an important evaluation of one's social abilities and induced a mind-set of seeking simple (vs. more complex) reasoning strategies. This goal-based, purely motivational manipulation affected mainly the reliance on category consistent (vs. inconsistent) information, whereas the timing of the presentation of the diagnostic relation was relatively less affected.

Thus, in this paper we aimed to examine, whether NFC induced via time pressure and goal activation, influences performance in basic attentional tasks that involve executive control, i.e., the Stroop task (1935) and the Social Category Switching Tasks (SCST, Marzecová, Bukowski, Boros, Correa, Lupiáñez, \& Wodniecka, 2013). We focus on these two tasks as, on the one hand, they both required the ability to inhibit dominant or prepotent responses, on the other hand, they differ in complexity, difficulty and type of stimuli used (words vs faces). Thus, we may gain an insight in executive control processes when performing cognitive tasks differed also in content (socially relevant vs. irrelevant). We predicted that elevated NFC will be related 
to more efficient performance in both tasks. Those effects should be more pronounced in the Stroop task than SCST, as the latter is more complex. This expectation is consistent with previous findings showing that individuals with a high NFC display a more efficient selectivity in the absence of load than do those with a low NFC (Kossowska, 2007b). However, even though high (vs. low) NFC individuals tend to perform better on a task involving selective attention, they perform more poorly on the same task than their low NFC counterparts, once the performers' resources are additionally taxed by apparent fatigue (Kossowska, $2007 b$ ). In addition, we hypothesized that time pressure and goal activation manipulations of NFC will influence performance in the same direction, that is, consistently with the first prediction, induced NFC levels will facilitate performance in tasks measuring basic executive functions of attention. Previous results showed impaired performance after induction of high NFC, but measuring more complex, integrative cognitive functions involved in reasoning about social relations (Bukowski et al., 2013). However, in this study we measure performance in basic attentional tasks, in which closure motives might enhance performance by the active maintenance of a single task goal in the face of distraction (Altamirano, Miyake, \& Whitmer, 2010).

\section{Method}

\section{Participants}

One hundred female ${ }^{1}$ and 11 male students participated in the study (aged $19-37, M=21.87$; $S D=$ 2.56 , one participant did not provide information concerning her age). Data from one subjects was excluded from the analyses because of the lack of experimental manipulation. Data from two subjects in the Stroop task were not coded, due to equipment malfunction. All of the participants were university students from Krakow, Poland. They were recruited through university classes or participant pool. Subjects recruited through classes received course credits while subjects from the pool were paid 5 euros.

\section{Procedure}

Participants were told that they were to participate in a study on individual differences in information processing. Each subject was seated in front of a computer screen and asked to follow experimenter instructions. Procedure included completing the two computer-based experimental tasks: color-naming Stroop task (Stroop, 1935) and Social Category Switching Task (Marzecová et al., 2013). Participants received an experimental manipulation inducing high or low NFC through time pressure or goal activation.

Time-pressure manipulation of NFC. In the time pressure condition participants were told that there is maximum time limit for performing two experimental tasks and that they would not be informed about the time left for the tasks. However, participants were warned that if they exceeded this interval they would be required to take another task. They were advised to perform experimental tasks as quickly as possible. In the no time pressure condition participants were informed that there is a minimal time requirement for the tasks and if they did not reach this minimal interval they just end the procedure. Accordingly, they were asked to complete experimental tasks as carefully as possible.

Goal-based manipulation of NFC. In both condition of goal-based manipulation participants were informed that all experimental tasks were designed to assess one facet of their cognitive performance. In high NFC condition instruction stated that research in the field of cognitive psychology clearly showed that "people with high levels of $I Q$ respond quickly and do not analyze given information deeply. Thus, try to respond quickly in the subsequent tasks. Work as quickly as you can". While participants in low NFC condition were given an instruction informing that cognitive psychology research demonstrated that "people with high levels of IQ work carefully and analyze incoming information thoroughly, even if it takes them more time. Thus, try to respond carefully in the subsequent tasks. Work as accurately as you can, do not worry about the time."

Color-naming Stroop task. Computerized version of color-naming Stroop task (1935) consisted of series of color words. The color of a presented stimulus word could be either congruent or incongruent with its semantic meaning. Participants were instructed to name the ink color of a presented word and to neglect its meaning. Subjects responded to each stimulus using computer keyboard (in a practice block they were asked to memorize which key represent respective color). The experimental trials were presented in a fixed random order. After 24 practice trials, participants were given 144 experimental trials. In each trial stimulus word was presented for $200 \mathrm{~ms}$; the maximum time for response was restricted to $2200 \mathrm{~ms}$. In practice block stimuli were presented until participant responded to it. The $2 / 3$ of stimuli were the congruent ones while remaining 1/3 incongruent ones. Previous studies (Tzelgov, Henik, \& Berger, 1992; West \& Alain, 2000) demonstrated that as the proportion of congruent stimuli increases, the prolonged response latency for incongruent stimuli (i.e. Stroop effect) increases. Despite the debate concerning the specific mechanism of this effect (e.g. Blais \& Bunge, 2010; Schmidt \& Besner, 2008) there is no doubt that task with small proportion of incongruent elicit more conflict. Thus, the task is more difficult for participants and this potentially protect against the ceiling effect. Stimuli were presented using DMDX software (Forster \& Forster, 2003).

Social Category Switching Task (SCST) tests the ability of switching between two social categories and was previously used to assess differences in cognitive flexibility (Marzecová et al., 2013). In this task participants categorised pictures of human faces, according to one of two social categories: gender (female vs. male) or age (young vs. old). Eight black and white photographs,

\footnotetext{
${ }^{1}$ It is worth noticing that the participant sample was largely gender biased, but some studies demonstrated that there are no gender differences in
} performing such elementary task as the Stroop task or switching tasks (Li, Zhang, Duann, Yan, Sinha, \& Mazure, 2009) 
depicting a young female, an old female, a young male, and an old male (two pictures for each category), were selected from The Center for Vital Longevity Face Database (Minear \& Park, 2004). All of the photographs were placed in the centre of the screen and had the same, following properties: $7.41 \mathrm{~cm}$ width and $6.74 \mathrm{~cm}$ height. A cue in the form of a coloured frame (green or purple) was presented to inform participants about which task (either gender or age categorisation) to perform. The colour - task combination was counterbalanced. The task was programmed in E-prime software (Schneider, Eschman, \& Zuccolotto, 2002).

Each trial in the procedure started with the presentation of a fixation cross for $1000 \mathrm{~ms}$. Afterwards, the target picture, framed with either the green or the purple coloured frame, was presented in the middle of the screen. The cue and the target remained on the screen until the participant responded, or for a maximum duration of 3000 ms. After incorrect responses, a beep was presented and the next trial followed after $1500 \mathrm{~ms}$. Participants responded using both hands. The " $\mathrm{z}$ " and " $\mathrm{m}$ " keys were used to respond to the gender task, whereas the age task was performed was performed with " $\mathrm{x}$ " and " $\mathrm{n}$ " keys. The matching of keys to category exemplars was counterbalanced across participants. Participants were given written instructions on the screen explaining the matching of keys and the tasks. The task contained with 8 practice trials, followed by four experimental blocks consisting of 80 trials each. The stimuli were presented in a random order to each participant.

\section{Results}

\section{Stroop Task}

In order to calculate the results for reaction time latencies, the incorrect as well as missed responses were excluded from further analyses (18,7\%). Moreover participants with accuracy lower than the chance level (in this case $25 \%$ ) either for congruent or incongruent stimuli were excluded from the sample (eleven participants). Thus, in statistical analyzes data from 98 participants remained (47 subjects in goal activation condition, 46 females; and 51 in time-pressure condition, 42 females).

First we checked the overall performance on the Stroop task, comparing the reaction times for congruent ( $M=685.99 \mathrm{~ms})$ to incongruent $(M=829.84 \mathrm{~ms})$ trials, and verifying the classic Stroop effect $(\mathrm{t}(97)=-14.67, p<$ $.001)$. The same pattern of results was found for accuracy scores - participants performed the task more accurately in congruent $(M=88 \%)$ than in incongruent $(M=78 \%)$ trials $(\mathrm{t}(97)=8.07, p<.001)$.

Further, we conducted a repeated measure ANOVA on response times with one within-subject factors: Congruency (Congruent vs. Incongruent trials), and one between-subjects factor, NFC level (high vs. low) for each of the two NFC manipulations separately (time pressure and goal activation). For the goal manipulation, the effect of NFC level manipulations turned out to be marginally significant $\left(F(1,45)=3.62, p=.064, \eta_{p}^{2}=.074\right)$, We did not find significant interaction effect between trial congruence and NFC level $\left(F(1,45)=1.84, p=.181, \eta_{p}^{2}=.039\right)$. The same analyzes were repeated for the accuracy as dependent variable. None of the effects reached statistical significance. For time-pressure manipulations, we found the main effect of NFC level $\left(F(1,49)=7.89, p<.01, \eta^{2}=.139\right)$, with participants in high NFC condition performing task faster than subjects in low NFC condition (congruent trials: $\mathrm{M}_{\text {high }}$ $=629.62 \mathrm{~ms}, \mathrm{M}_{\text {low }}=740.57 \mathrm{~ms}$; incongruent trials: $\mathrm{M}_{\text {high }}$ $=716.81 \mathrm{~ms}, \mathrm{M}_{\text {low }}=869.45 \mathrm{~ms}$, for low and high NFC subjects, respectively). This effect however was qualified by marginally significant 2 -way interaction between trial congruence and NFC level $\left(F(1,49)=3.09, p=.085, \eta_{p}^{2}=\right.$ $.059)$. The same analyzes were repeated for the accuracy $(\%$ of accurate responses) as a dependent variable. None of the effects reached statistical significance $(F<1)$.

Overall analyzes. Based on the theory, we expected that both types of NFC manipulation (i.e., time pressure and goal activation) will influence the task performance in the same direction. Moreover, the analyses conducted separately for each type of NFC manipulation did not provide any significant results for low vs. high NFC participants. Thus we decided to analyze data obtained from both groups with different manipulations together to increase the test power.

Response times to congruent and incongruent trials were analyzed in a 2 (Congruency: Congruent vs. Incongruent; within participants) x 2 (NFC level: high vs. low, between participants) x 2 (Type of NFC manipulation: time pressure vs. goal activation, between participants) mixed model ANOVA. We found a significant main effect of the NFC level $\left(F(1,94)=10.13, p=.002, \eta_{p}^{2}=.097\right)$. This main effect was qualified by a 2 -way interaction effect between NFC level and congruence of the trial $(F(1,94)$ $=4.70, p=.033, \eta_{p}^{2}=.048$ ), showing a less pronounced difference in reaction times for congruent and incongruent trials in high vs. low NFC participants. In other words, the Stroop effect was weaker in conditions of induced high NFC (difference in means between congruent and incongruent trials $=123,46 \mathrm{~ms}$ ) than in low NFC (difference in means between congruent and incongruent trials $=166,26 \mathrm{~ms})$. We did not find a three-way interaction effect 2 (Congruency) $\mathrm{x}$ 2 (induced NFC level) x 2 (Type of NFC manipulation) for reaction times.

The same analysis was repeated for the accuracy (\%) as dependent variable. We found marginal main effects of NFC level manipulations $\left(F(1,94)=2.65, p=.11, \eta_{p}^{2}=\right.$ $.027)$ with more accurate responses of participants in high NFC than low NFC condition $\left(\mathrm{M}_{\text {high }}=86 \%, \mathrm{M}_{\text {low }}=81 \%\right)$.

\section{Social Category Switching Task}

Erroneous and missed responses, RTs shorter than 200 and higher than $2000 \mathrm{~ms}$, as well as RTs from the first trial of each block were excluded from the analysis of response times (altogether 10,7\%). Two participants were excluded from final analyses due to a very low percentage of accurate responses, which might be related to a random response tendency or misunderstanding of the instructions (accuracy rates below $44 \%$ of correct responses). Firstly, we checked the overall performance in the SCST and found the expected main effect of switching, that is longer reaction 
times for switch $(M=1033 \mathrm{~ms})$ than non-switch trials $(M=$ $893 \mathrm{~ms})\left(F(1,108)=338.4, p<.001, \eta_{p}^{2}=.76\right)$.

Further, we conducted a repeated measure ANOVA with two within-subject factors: Task Switch (task switch vs. no switch) and Repetition Type (alternation vs. complete repetition vs. partial repetition of stimuli), and one betweensubjects factor, NFC level (high vs. low) for each of the two NFC manipulations separately (time pressure and goal activation). For the goal manipulation, we did not find a three-way interaction $(F<1)$ and we found a tendency in the switch $\mathrm{x}$ NFC level interaction $(F(1,52)=2.81, p=.099$, $\left.\eta_{p}^{2}=.05\right)$, indicating a worse performance after low NFC induction in switch (vs. non-switch) trials. For the time pressure manipulation we did neither find a significant switch $\mathrm{x}$ repetition type $\mathrm{x}$ NFC level interaction $(F(1,52)=$ $\left.1.76, p=.18, \eta^{2}=.03\right)$, nor a two way switch $\mathrm{x}$ NFC level interaction $\left(F(1,52)=1.98, p=.16, \eta_{p}^{2}=.04\right)$. However, both interactions revealed a weak statistical trend going in the same direction as in the goal manipulation (i.e. worse performance following low NFC induction).

Since the effects for both types of manipulations were analogous, in order to check for the role of a specific NFC manipulation and to compare it with the overall effect of NFC level, we conducted one repeated measure ANOVA with two within-subject factors: Task Switch (task switch vs. no switch) and Repetition Type (alternation vs. complete repetition vs. partial repetition of stimuli), and two between-subjects factors: Type of NFC manipulation (time pressure vs. goal activation) and NFC level (high vs. low). We found a marginal main effect of the NFC level manipulation, showing overall faster reaction times in the switching task after high NFC $(M=938 \mathrm{~ms})$ relatively to low NFC induction $(M=988 \mathrm{~ms})(F(1,105)=3.49, p=.06$, $\left.\eta_{p}^{2}=.04\right)$.

We did not find a four-way interaction effect $(F<$ 1). However, a two-way repeated measure ANOVA revealed a significant interaction between Task Switch and NFC level $\left(F(1,105)=4.78, p=.031, \eta_{p}^{2}=.04\right)$. Pairwise comparisons (Sidak) showed that after situationally induced high (vs. low) NFC participants reacted faster in trials in which a category switch was required $\left(M_{\text {high }} \mathrm{NFC}=999 \mathrm{~ms}, \mathrm{M}_{\text {low }}\right.$ $\left.\mathrm{NFC}=1067 \mathrm{~ms} ; F(1,105)=4.47, p=.037, \eta_{p}^{2}=.04\right)$. The same comparison for non-switch trials was not significant $\left(\mathrm{M}_{\text {high }} \mathrm{NFC}=876 \mathrm{~ms}, M_{\text {low }} \mathrm{NFC}=911 \mathrm{~ms} ; F(1,105)=\right.$ $2.04, p=.16, \eta_{p}^{2}=.02$ ). In sum, reaction times analyses revealed smaller switching costs after high than low NFC manipulations. We have also found a non-significant effect for the switch $\mathrm{x}$ NFC manipulation condition $(F(1,105)=$ $2.70, p=.10, \eta_{p}^{2}=.02$ ).

We have also performed analogous analyses for the accuracy measure. The four-way, Task Switch x Repetition Type $\mathrm{x}$ Type of NFC manipulation x NFC level did not yield significance $(F<1)$. However, a significant interaction between Repetition Type and NFC level was observed $\left(F(1,104)=3.4, p=.035, \eta_{p}^{2}=.03\right)$, which indicated that in high NFC conditions participants were less accurate in conditions that involved stimulus change (e.g. change from a young woman to an old woman) in comparison to conditions not involving stimulus change. Pairwise comparisons revealed that this effect was mainly pronounced when the stimulus changed completely (complete alternation condition, $M=.90, S D=.01$ ) or partially (partial repetition, $M=.90, S D=.01)$ relatively to the complete repetition condition, when the stimulus remained the same (complete repetition, $M=.93, S D=.01)(F(1,104)=12.44, p<.001$, $\left.\eta_{p}^{2}=.2\right)$. Participants in low NFC conditions did not show this pattern and responded on a comparable level to all types of repetitions $\left(F(1,104)=1.87, p=.16, \eta_{p}^{2}=.03\right)$. In other words, the accuracy of responses after high NFC induction was more sensitive to the type of stimulus repetition, in a way, that any change in the stimulus type evoked more erroneous reactions, whereas after low NFC induction the accuracy level was more stable across task conditions.

\section{Discussion}

Several studies have provided evidence for the conclusion that NFC as individual characteristic is associated with better performance in tasks involved executive control functions (Kossowska, 2007 a, b; Kossowska et al., 2010). However, what is missing in those studies is a consideration of state-dependent influences. Thus, in order to validate the previous findings, we focused on examining the effect of time pressure and goal activation on executive control, as research demonstrated that these two types of NFC manipulation influence different aspects of knowledge formation process (Bukowski et al., 2013). However, we expected that these two types of NFC manipulations would induce a similar pattern of responses in Stroop task and SCST for high vs. low NFC conditions. Thus, taking all data into account (from time pressure and goal activation manipulations) we focused only on the differences between the levels of NFC.

Consistently with our hypothesis, indeed we found that NFC, evoked by both, time pressure and goal activation, improved performance on two tasks that involve executive control. Generally speaking, in the Stroop task high (vs. low) NFC participants demonstrated weaker Stroop effect and in the SCST, they also exhibited smaller switching costs. In the light of these results we may suggest that high (vs. low) NFC is associated with higher ability to handle with interference. It is important to notice that those results were obtained only when reaction times were taken into account. However, the results from the switching task reveal an interesting possibility that there are some hidden costs of increased efficiency of induced high NFC, namely the effectiveness of performance (response accuracy in particular stimulus repetition conditions) decreased in those task conditions, which involved stimulus change. This result resembles a trade-off pattern, in which hight NFC participants compensate impaired processing efficiency with additional effort devoted to the task, yet the accuracy results do not encompass the category switching variable, which is central for the results obtained for reaction time latencies. Additionally, in the Stroop task we did not find any confirmation for the trade-off hypothesis. Therefore the interpretation of higher costs of increased efficiency amongst high NFC participants should be considered with caution and requires further research. 
Although, we are aware that the way we treat two types of manipulations could rise some controversies, the results we obtained are important not only because they are validating the previous findings. They also indicated the "bright side" of NFC. Many studies have proven the tendency of high NFC people to become stuck in a certain inflexible mind-set. Although mental inflexibility is often disadvantageous (especially when the situation calls for rapidly shifting between goals), it can be advantageous when successful performance requires active maintenance of a particular goal despite distracting stimuli or competing goals. In such situations, such mental "stickiness" of individuals high in NFC may help to prevent that goal from easily slipping out of working memory. Previous research showed improved selective attention under stress, due to an enhanced selectivity to task-relevant attributes because of reduced utilization of task-irrelevant attributes (Chajut \& Algom, 2003; Kossowska, 2007a, b). In a similar vein, our research showed that when external demands in a form of time pressure are present or the subjective relevance of cognitive closure goals is high, then cognitive conflict resolution might be more efficient due to a more selective attentional focus on filtering out the irrelevant information. At the same time, it could be predicted that there is an optimal level of time related stress for performance on cognitive tasks, which can be depicted by a curve-linear relation, that is when the external demands (e.g. time pressure, task complexity) increase, then the efficiency of using a simple filtering strategy might be reduced (Zivnuska, Kiewitz, Hochwater, Perrewe, \& Zellars, 2002; Kossowska 2007b).

Interestingly, our study revealed analogous effects of both closure manipulations on performance in tasks assessing executive control. However, it is worth emphasizing the differences between performance of high NFC people in both executive tasks. The accuracy of responses after high NFC induction in SCST was more sensitive to the type of stimulus repetition, in a way, that any change in the stimulus type evoked more erroneous reactions, whereas after low NFC induction the accuracy level was more stable across task conditions. This result might suggest that there were some costs of quicker reaction times in switch and non-switch trials in a form of less accurate performance but only when the stimuli changed to a greater degree, introducing in a certain way more noise to the task. In other words, any changes in the goal shifting rules resulted in decreased performance of high NFC individuals. One explanation of those results may be linked to sensitivity of high NFC people to increased difficulty of the task. As it was previously demonstrated even though high (vs. low) need for closure individuals tend to perform better on a tasks involving selective attention, they perform more poorly on the same task than their low need for closure counterparts, once the performers' resources are additionally taxed by difficulties (Kossowska, 2007b). In sum, the results obtained in the SCST show that although elevated NFC leads to smaller switching costs (related to overall faster reaction times), in more complex task conditions, that involve rule changes, this facilitation is at the cost of diminished response accuracy.
An alternative explanation of this result stresses the dissociation between two goal-neglect tasks, which were used in the study. Although both tasks were designed to measure executive control, one emphasized goal maintenance (Stroop), the other emphasized goal shifting (SCST). Thus these two tasks differ in degree to which people's cognitive processing is geared toward flexibility (goal shifting) or stability (goal maintenance). And of course, cognitive processing of high (vs. low) NFC individuals may inherently be geared more toward goal maintenance than toward goal shifting. Thus, it could be expected that when goal shifting rules become more difficult, then the advantage stemming from higher levels of NFC might turn into a disadvantage, leading to more erroneous or biased responses. This issue calls for examination in future research.

Relating the results obtained in our present study to some previous findings on the impact of different closure manipulations on cognitive performance, it might be stated, that there are two routes to closure which depending on the type of task requirements can have uniform or diverse effects on performance. If the task requires the execution of clear, non-ambiguous rules (e.g. name the color of the font and ignore the meaning of the word), then even if this activity involves effortful and systematic processing, then high levels of NFC might help to execute it. However, if the task requires paying attention to more complex and multiple rules (e.g. integrate various sources of information into a coherent mental model) then high levels of NFC can decrease performance (Bukowski et al., 2013; Bukowski, Sędek, Kossowska, \& Trejtowicz, 2012; Kossowska, $2007 a, b)$. Another important difference between this research and some previous studies is that the effects obtained here mainly refer to reaction times latencies in reacting to relatively easy categorizable stimuli (color, gender, age), whereas some previous studies showed the biasing nature of high NFC on the focus and preference for background knowledge consistent information (Kruglanski et al., 2009). An intriguing and couter-intuitive general conclusion that seems to follow from this research is that high NFC levels might only lead to its adverse effects on information processing, when the environment is complex or unstructured leading to the preference of knowledge consistent structures but when the task rules are structured and well-defined then high levels of closure can boost performance also when managing with cognitive conflict and inconsistency is involved. Therefore, in future research, examining the joint impact of the type of closure induction and complexity of task rules on performance seems an interesting line to follow.

\section{References}

Altamirano, L. J., Miyake, A., Whitmer, A. J. (2010). When Mental Inflexibility Facilitates Executive Control: Beneficial Side Effects of Ruminative Tendencies on Goal Maintenance. Psychological Science, 21, 1377-1382. doi:10.1177/0956797610381505

Amodio, D., Jost, J., Masters, S. \& Yee, C. (2008). Neurocognitive correlates of liberalism and conservatism. Nature Neuroscience, 10, 1246-1247. 
Bar-Tal, Y., Kishon-Rabin, L., \& Tabak, N. (1997). The effect of need and ability to achieve cognitive structuring on cognitive structuring. Journal of Personality and Social Psychology, 73, 1158-1176. doi:10.1037//0022-3514.73.6.1158

Blais, C., \& Bunge, S. (2010). Behavioral and Neural Evidence for Itemspecific Performance Monitoring. Journal of Cognitive Neuroscience, 22, 2758-2767.

Bukowski, M., Sędek, G., Kossowska, M., \& Trejtowicz, M. (2012). The impact of background category information on the creation of socia cliques: The role of need for cognitive closure and decisiveness. Polish Psychological Bulletin, 43, 12-19.

Bukowski, M., von Hecker, U., \& Kossowska, M. (2013). Motivational determinants of reasoning about social relations: The role of need for cognitive closure. Thinking and Reasoning, 19, 150-177. doi:10.108 $0 / 13546783.2012 .752407$

Chajut, E., \& Algom, D. (2003). Selective attention improves under stress: Implications for theories of social cognition. Journal of Personality and Social Psychology, 85, 231-248. doi:10.1037/00223514.85.2.231

Chiu, C., Morris, M. W., Hong, Y., \& Melon, T. (2000). Motivated cultural cognition: The impact of implicit cultural theories on dispositional attribution varies as a function of need for closure. Journal of Personality and Social Psychology, 78, 247-259.

Dijksterhuis, A., van Knippenberg, A., Kruglanski, A. W., \& Schaper, C. (1996). Motivated social cognition: Need for closure effects on memory and judgement. Journal of Experimental and Social Psychology, 32, 254-270.

Driscoll, D.M., Hamilton, D.L., \& Sorrentino, R.M. (1991). Uncertainty orientation and recall of person-descriptive information. Personality and Social Psychology Bulletin, 17, 494-500.

Ford, T. E., \& Kruglanski, A. W. (1995). Effects of epistemic motivations on the use of accessible constructs in social judgment. Personality and Social Psychology Bulletin, 21, 950-962.

Forster, K. I., \& Forster, J. C. (2003). DMDX: A Windows display program with millisecond accuracy. Behavior Research Methods, Instruments, and Computers, 35, 116-124.

Freund, T., Kruglanski, A. W., \& Shpitzajzen, A. (1985). The freezing and unfreezing of impressional primacy: Effects of the need for structure and the fear of invalidity. Personality and Social Psychology Bulletin $11,479-487$

Heaton, A. W., \& Kruglanski, A. W. (1991). Person perception by introverts and extroverst under time pressure: Effects of need for closure. Personality and Social Psychology Bulletin, 17, 161-165.

Inzlicht, M., McGregor, I., Hirsh, J. \& Ash, K. (2009). Neural markers of religious conviction. Psychological Science, 20, 385-392.

Jamieson, D. W., \& Zanna, M. P. (1989). Need for structure in attitude formation and expression. In A. Pratkanis, S. Breckler, \& A. G Greenwald ( Eds. ), Attitude structure and function ( pp. 46-68). Hillsdale, N J: Erlbaum.

Kossowska, M. (2007a). The role of cognitive inhibition in motivation toward closure. Personality and Individual Differences, 42, 11171126.

Kossowska, M. (2007b). Motivation toward closure and cognitive processes: An individual differences approach. Personality and Individual Differences, 43, 2149-2158.

Kossowska, M., Orehek, E., Kruglanski, A.W. (2010). Motivation toward closure and cognitive resources: An individual differences approach. In A. Gruszka, G. Mathews, and B. Szymura (Eds.), Handbook of individual differences in cognition: attention, memory and executive control (pp. 369-382). New York, NY US: Springer Science + Business Media.

Kruglanski, A. W. (2004). The psychology of closed mindedness. New York: Psychology Press.

Kruglanski, A. W., \& Freund, T. (1983). The freezing and un-freezing of lay-inferences: Effects of impressional primacy, ethnic stereotyping and numerical anchoring. Journal of Experimental Social Psychology, $19,448-468$

Kruglanski, A. W., Freund, T., \& Bar-Tal, D. (1996). Motivational effects in the mere-exposure paradigm. European Journal of Social Psychology, 26, 479-499.

Kruglanski, A. W., Dechesne, M., Orehek, E., \& Pierro, A. (2009). Three decades of lay epistemics: The why, how, and who of knowledge formation. European Review of Social Psychology, 20, 146-191. doi:10.1080/10463280902860037
Kruglanski, A. W., \& Webster, D. M. (1996). Motivated closing of the mind: "Seizing" and "Freezing". Psychological Review, 103, 263283.

Kruglanski, A. W., Webster, D. M., \& Klem, A. (1993). Motivated resistance and openness to persuasion in the presence or absence of prior information. Journal of Personality and Social Psychology, 65, 861-877.

Li, C., Zhang, S., Duann, J., Yan, P., Sinha, R., \& Mazure, C. (2009). Gender differences in cognitive control: An extended investigation of the stop signal task. Brain Imaging and Behavior, 3, 262-276. DOI 10.1007/s11682-009-9068-1

Marzecová, A., Bukowski, M., Boros, M., Correa, A., Lupiáñez, J., \& Wodniecka, Z. (2013). Tracing the bilingual advantage in cognitive control: The role of flexibility in temporal preparation and category switching. Journal of Cognitive Psychology, 25, 586-604.

Miller, E. K., \& Cohen, J. D. (2001). An integrative theory of prefrontal cortex function. Annual Review of Neuroscience, 24, 167-202.

Minear, M., \& Park, D. C. (2004). A lifespan database of adult facial stimuli. Behavior Research Methods, Instruments, \& Computers, 36(4), 630-633. doi:10.3758/BF03206543

Miyake, A., Friedman, N., Emerson, M., Witzki, A., \& Howerter, A. (2000). The unity and diversity of executive functions and their contribution to complex "frontal lobe" tasks: a latent variable analysis. Cognitive Psychology, 41, 49-100.

Richter, L., \& Kruglanski, A. W. (1998). Seizing on the latest. Motivationally driven recency effects in impression formation. Journal of Experimental Social Psychology, 34, 313-329.

Roets, A., \& Van Hiel, A. (2011). Impaired performance as a source of reduced energy investment in judgment under stressors. (European) Journal of Cognitive Psychology, 23, 625-632.

Roets, A., Van Hiel, A., Cornelis, I., \& Soetens, B. (2008). Determinants of task performance and invested effort: A need for closure by ability interaction analysis. Personality and Social Psychology Bulletin, 34, 779-792.

Schmidt, J. R., \& Besner, D. (2008). The Stroop Effect: Why proportion Congruent Has Nothing to Do With Congruency and Everything to Do With Contingency. Journal of Experimental Psychology. Learning, Memory \& Cognition, 34(3), 514-523. doi:10.1037/02787393.34.3.514

Schneider, W., Eschman, A., \& Zuccolotto, A. (2002). E-Prime User's Guide. Pittsburgh: Psychology Software Tools, Inc.

Stroop, J.R. (1935). Studies on interference in serial verbal reactions. Journal of Experimental Psychology, 18, 643-662. Retrieved from http://psychclassics.yorku.ca/Stroop

Tzelgov, J., Henik, A., \& Berger, J. (1992). Controlling Stroop effects by manipulating expectations for color words. Memory \& Cognition, 20, 727-735.

Webster, D. M. (1993a). Motivated augmentation and reduction of the overattribution bias. Journal of Personality and Social Psychology, $65,261-271$

Webster, D. M. (1993b). Groups under the influence: Need for closure effects on information sharing in decision making groups. Unpublished doctoral dissertation. University of Maryland, College Park.

Webster, D. M., \& Kruglanski, A. W. (1994). Individual differences in need for cognitive closure. Journal of Personality and Social Psychology, 67, 1049-1062.

Webster, D. M., Richter, L., \& Kruglanski, A. W. (1996). On leaping to conclusions when feeling tired: Mental fatigue effects on impressional primacy. Journal of Experimental Social Psychology, 32, 181-195.

West, R., \& Alain, C. (2000). Effects of task context and fluctuations of attention on neural activity supporting performance of the Stroop task. Brain Research, 873, 102-111.

Zivnuska, S., Kiewitz, C., Hochwater, W. A., Perrewe, P. L., \& Zellars, K. L. (2002). What is too much or too little? The curvilinear effects of job tension on turnover intent, value attainment, and job satisfaction. Journal of Applied Social Psychology, 32, 1344-1360. doi:10.1111/j.1559-1816.2002.tb01440.x 